ARTICLE

\title{
Structural basis for nuclear import selectivity of pioneer transcription factor SOX2
}

Bikshapathi Jagga', Megan Edwards², Miriam Pagin ${ }^{3}$, Kylie M. Wagstaff4, David Aragão (1) 5, Noelia Roman?', Jeffrey D. Nanson (1D ${ }^{6}$, Shane R. Raidal (10 ${ }^{7}$, Nicole Dominado (1) ${ }^{8}$, Murray Stewart ${ }^{9}$, David A. Jans (10) ${ }^{4}$, Gary R. Hime (iD ${ }^{8}$, Silvia K. Nicolis ${ }^{3}$, Christopher F. Basler (iD ${ }^{2} \&$ Jade K. Forwood ${ }^{1 \times}$

SOX (SRY-related HMG-box) transcription factors perform critical functions in development and cell differentiation. These roles depend on precise nuclear trafficking, with mutations in the nuclear targeting regions causing developmental diseases and a range of cancers. SOX protein nuclear localization is proposed to be mediated by two nuclear localization signals (NLSs) positioned within the extremities of the DNA-binding HMG-box domain and, although mutations within either cause disease, the mechanistic basis has remained unclear. Unexpectedly, we find here that these two distantly positioned NLSs of SOX2 contribute to a contiguous interface spanning 9 of the 10 ARM domains on the nuclear import adapter $I M P \alpha 3$. We identify key binding determinants and show this interface is critical for neural stem cell maintenance and for Drosophila development. Moreover, we identify a structural basis for the preference of SOX2 binding to IMP $\alpha 3$. In addition to defining the structural basis for SOX protein localization, these results provide a platform for understanding how mutations and post-translational modifications within these regions may modulate nuclear localization and result in clinical disease, and also how other proteins containing multiple NLSs may bind IMP $\alpha$ through an extended recognition interface.

\footnotetext{
${ }^{1}$ School of Biomedical Sciences, Charles Sturt University, Wagga Wagga, NSW 2678, Australia. ${ }^{2}$ Center for Microbial Pathogenesis, Institute for Biomedical Sciences, Georgia State University, Atlanta, GA 30303, USA. ${ }^{3}$ Department of Biotechnology and Biosciences, University of Milano-Bicocca, piazza della Scienza 2, 20126 Milano, Italy. ${ }^{4}$ Biomedicine Discovery Institute, Monash University, Clayton, VIC 3800, Australia. ${ }^{5}$ Diamond Light Source, Harwell Science and Innovation Campus, Didcot OX11 ODE, UK. ${ }^{6}$ School of Chemistry and Molecular Biosciences, Institute for Molecular Bioscience and Australian Infectious Diseases Research Centre, University of Queensland, Brisbane, QLD 4072, Australia. ${ }^{7}$ School of Animal and Veterinary Sciences, Charles Sturt University, Wagga Wagga, NSW 2678, Australia. ${ }^{8}$ Department of Anatomy and Neuroscience, University of Melbourne, Parkville, VIC 3010, Australia. ${ }^{9}$ MRC

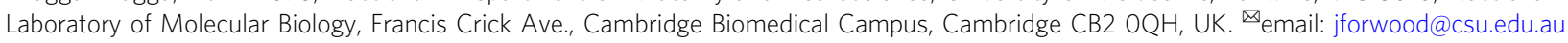


T he human SOX (sex-determining region Y (SRY)-related HMG-box) family of transcription factors comprises 20 members that play critical roles in organogenesis, stem cell maintenance, and cancer progression ${ }^{1}$. These proteins can act as both tumor suppressors or activators depending on the cellular environment ${ }^{2}$. SOX2 is essential for the neural development, and the self-renewal of undifferentiated embryonic and neural stem cells. It is spatially and temporally expressed during development; initial expression occurs at the preimplantation embryo stage, with restriction to the blastocyst inner cell mass and epiblast ${ }^{3}$. Expression is also found in the anterior ectoderm to facilitate formation of the neuroectoderm and anterior surface ectoderm ${ }^{4}$. During later stages of development, SOX2 expression is found in the primitive foregut endoderm ${ }^{3,5}$. SOX2 is one of the key factors required to convert somatic cells into induced pluripotent stem cells (PSCs), and in concert with Nanog and Oct4, plays a central role in embryonic stem cell maintenance ${ }^{3,6}$. These roles require precise and timely localization of SOX2 to the nucleus. To gain access to the nucleus, SOX proteins harbor two NLSs, located distally at the $\mathrm{N}$ - and C-terminus of the DNA-binding domain $^{7-15}$. This arrangement is conserved across all SOX family members (Fig. 1), and mutations within these regions can impair nuclear localization, cause severe developmental disease, and are associated with poor prognosis in cancer, ${ }^{9-19}$ (see also "Abstract" section).

The localization of proteins to the nucleus via the classical import pathway is an active process and requires a cargo bearing an NLS to be recognized by importin- $\alpha$ (IMP $\alpha)^{20}$. The cargo: IMP $\alpha$ complex is transported through the nuclear pore complex by importin- $\beta$ (IMP $\beta)^{21,22}$ to the nucleus, where it is disassembled by RanGTP. Humans harbor seven IMPa isoforms (IMPa1-7), each containing an N-terminal IMP $\beta$-binding domain (residues 1-70) and a C-terminal NLS-binding domain constructed from ten armadillo (ARM) repeats (residues 70-500). Many nuclear import cargos exhibit specificity toward these isoforms ${ }^{14,23}$. For example, RCC1, the Ran nucleotide exchange factor that establishes the directionality of nuclear transport, and HIV-1 integrase, responsible for integrating the HIV-1 genome into the DNA of an infected cell, bind specifically to IMPa3 (refs. ${ }^{24,25}$ ); STAT1, a signaling molecule in the innate immune system, binds specifically to the convex C-terminal surface of IMPa5-7 (ref. ${ }^{23,26}$ ). Ebola VP24 binds specifically to IMPa5 to selectively compete with the nuclear import of phosphorylated STAT1 (ref. ${ }^{27}$ ). SOX proteins show remarkable isoform specificity mechanisms, and strikingly undertake isoform-specific switching during differentiation ${ }^{14}$. For example, neural differentiation of embryonic stem cells is mediated by IMPa isoform switching such that Oct $3 / 4$ is driven to the nucleus by IMPal in undifferentiated stem cells; however, during neural development, upregulation of IMPa3/5 mediates SOX2/Brn2 nuclear import and neural differentiation ${ }^{14}$. The molecular basis for this specificity is unclear, and understanding IMPa specificity is complicated by the seven human IMPa isoforms all containing highly conserved NLS-binding regions.

\section{Results}

SOX2 NLSs are bound by IMPa3 through a contiguous interface. To better understand the mechanisms of how critical signaling regions in SOX proteins interact with nuclear import receptors to drive nuclear transport, we crystallized the HMG domain of SOX2 (comprising residues 39-127) in complex with different IMPa isoforms. The $2.3 \AA$ A resolution structure of IMPa 3 bound to SOX2 (see Supplementary Table 1 for crystallographic statistics; other isoforms are discussed below), enabled the entire HMG-box domain and NLS regions of SOX2 to be reliably traced from the electron density. SOX2 bound IMPa3 through an

a
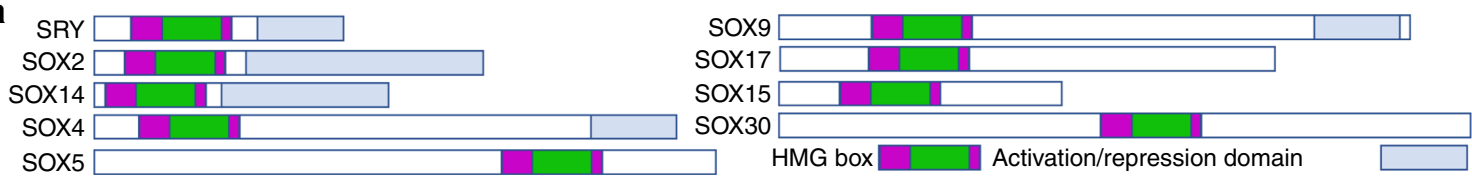

b

B1 hSOX1

B1 hSOX2

B1 hSOX3

B2 hsox14

B2 hsOX21

C hSOX 4

C hSOX11

C hSOX12

D hSOX 5

D hSOX 6

D hSOX13

E hSOX8

E hSOX 9

E hSOX10

F hSOX 7

F hSOX17

F hSOX18

G hSOX15

$\mathrm{H} \quad \mathrm{hSOX} 30$
Minor-site NLS NLS1

Unique IMP $\alpha 3$ specificity binding region
Major-site NLS NLS2

${ }^{58}$ DRVKRPMNAF IVWSRDQRRKMALENPRMRNSEISKQLGYQWKMLTEAEKWPFFQEAQKLQAMHREKY PNYKYRPRRKAKMLP139

${ }^{49}$ DRVKRPMNAFMVWSRGQRRKMAQENPKMHNSEISKRLGAEWKVMSEAEKRPFIDEAKRLRALHMKEHPDYKYRPRRKTKTLL130

${ }^{39}$ DRVKRPMNAFMVWSRGQRRKMAQENPKMHNSEISKRLGAEWKLLSETEKRPF IDEAKRLRALHMKEHPDYKYRPRRKTKTLM ${ }^{120}$

${ }^{137}$ DRVKRPMNAFMVWSRGQRRKMALENPKMHNSEISKRLGADWKLLTDAEKRPFIDEAKRLRAVHMKEYPDYKYRPRRKTKTLL 218

${ }^{6}$ DH I KRPMNAFMVWSRGQRRKMAQENPKMHNSEISKRLGAEWKLLSEAEKRPYIDEAKRLRAQHMKEHPDYKYRPRRKPKNLL ${ }^{87}$

${ }^{6}$ DHVKRPMNAFMVWSRAQRRKMAQENPKMHNSEISKRLGAEWKLLTESEKRPFIDEAKRLRAMHMKEHPDYKYRPRRKPKTLL ${ }^{87}$

${ }^{57}$ GHIKRPMNAFMVWSQ IERRKIMEQS PDMHNAEISKRLGKRWKLLKDSDKIPFIREAERLRLKHMADY PDYKYRPRKKVKSGN 138

${ }^{47}$ GHIKRPMNAFMVWSKIERRKIMEQSPDMHNAEISKRLGKRWKMLKDSEKIPFIREAERLRLKHMADY PDYKYRPRKKPKMDP ${ }^{128}$

${ }^{38}$ GHI KRPMNA FMVWSQHERRKIMDQWPDMHNAE ISKRLGRRWQLLQDSEKI PFVREAERLRLKHMADYPDYKYRPRKKSKGAP 119

${ }^{554} \mathrm{PHI}$ KRPMNAFMVWAKDERRKILQAFPDMHNSNISKILGSRWKAMTNLEKQPYYEEQARLSKQHLEKYPDYKYKPRPKRTCLV635

${ }^{619} \mathrm{PHIKRPMNAFMVWAKDERRKILQAFPDMHNSNI} \mathrm{SKILGSRWKSMSNQEKQPYYEEQARLSKIHLEKY} \mathrm{PNYKYKPRPKRTCIV700}$ ${ }^{422}$ SHIKRPMNAFMVWAKDERRKILQAFPDMHNSS ISKILGSRWKSMTNQEKQPYYEEQARLSRQHLEKYPDYKYKPRPKRTCIV503 ${ }^{100}$ PHVKRPMNAFMVWAQAARRKLADQYPHLHNAELSKTLGKLWRLLSESEKRPFVEEAERLRVQHKKDHPDYKYQPRRRKSAKA ${ }^{181}$ ${ }^{103}$ PHVKRPMNAFMVWAQAARRKLADQYPHLHNAELSKTLGKLWRLLNESEKRPFVEEAERLRVQHKKDHPDYKYQPRRRKSVKN 184 ${ }^{102}$ PHVKRPMNAFMVWAQAARRKLADQYPHLHNAELSKTLGKLWRLLNESDKRPFIEEAERLRMQHKKDHPDYKYQPRRRKNGKA ${ }^{183}$ ${ }^{43}$ SRIRRPMNAFMVWAKDERKRLAVQNPDLHNAELSKMLGKSWKALTLSQKRPYVDEAERLRLQHMQDY PNYKYRPRRKKQAKR ${ }^{124}$ ${ }^{66}$ SRIRRPMNAFMVWAKDERKRLAQQNPDLHNAELSKMLGKSWKALTLAEKRPFVEEAERLRVQHMQDHPNYKYRPRRRKQVKR ${ }^{147}$ ${ }^{83}$ SRIRRPMNAFMVWAKDERKRLAQQNPDLHNAVLSKMLGKAWKELNAAEKRPFVEEAERLRVQHLRDHPNYKYRPRRKKQARK ${ }^{164}$ ${ }^{47}$ EKVKRPMNAFMVWS SAQRRQMAQQNPKMHNSEISKRLGAQWKLLDEDEKRPFVEEAKRLRARHLRDYPDYKYRPRRKAKS SG ${ }^{128}$ 335 GHVKRPMNAFMVWAR I HRPALAKANPAANNAE I SVOLGLEWNKLSEEOKKPYYDEAOKI KEKHREEF PGWVYOPRPGKRKRF 416

Fig. 1 Domain organization within the SOX family. a The SOX family is comprised of 20 members and are grouped based on domain organization. A representative from each SOX group is shown. $\mathbf{b}$ Each SOX family member contains a highly conserved HMG domain (in green/pink), with NLSs positioned within the extremities of the HMG domain. The residues within SOX2 that form hydrogen bonds or salt bridges with IMP $\alpha 3$ in our crystal structure are highlighted (in pink). 
a

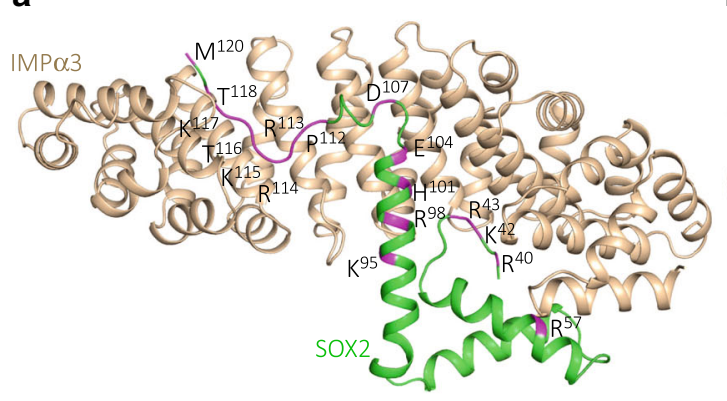

b

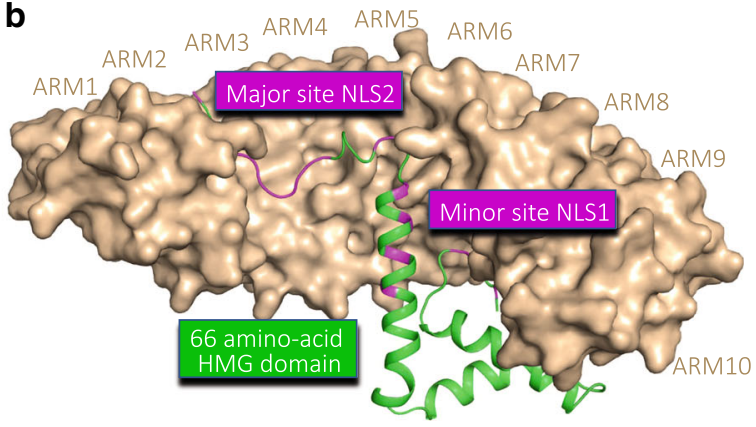

c

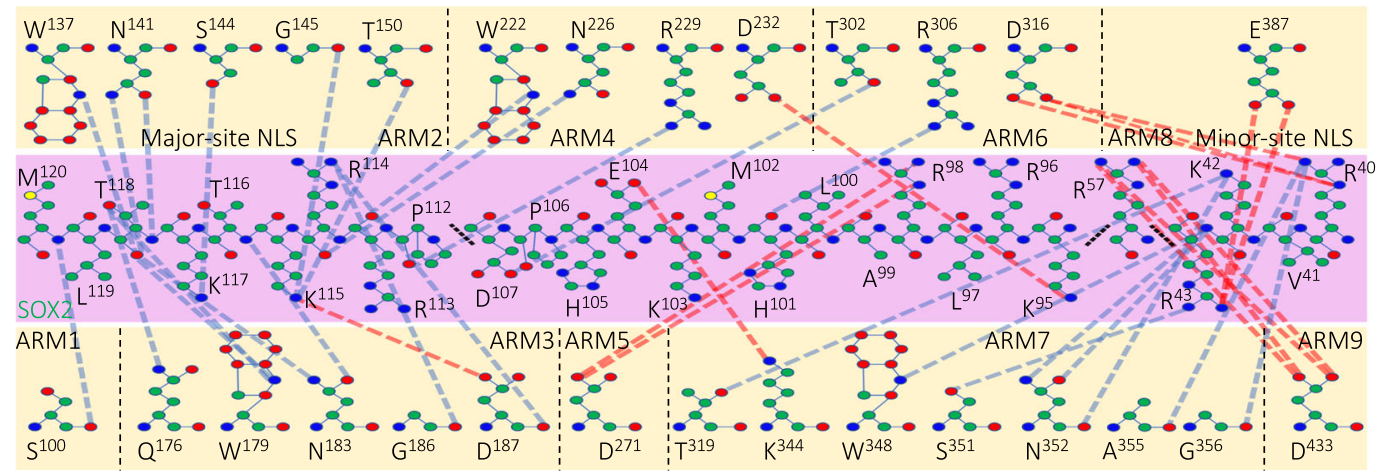

Fig. 2 The two distally positioned NLSs of SOX2 bind to IMP $\alpha 3$ as part of a single continuous interface. a Structure of IMP $\alpha 3$ (shown in light brown, cartoon/ribbon format) bound to SOX2 (shown in green, cartoon/ribbon format). Residues within SOX2 that form salt bridges or hydrogen bonds with IMP $\alpha 3$ are highlighted pink. b Structural overview of SOX2 binding IMP $\alpha 3$ (shown in surface view), highlighting how the two distantly positioned NLSs of SOX2 are in close proximity and bind at a single interface. c Detailed map of the residues in the interaction interface with hydrogen bonds shown in blue and salt bridge interactions in red. Interactions are also listed in Supplementary Tables 2-4.

extensive and contiguous interface across ARM domains 1-9 of IMPa3 (Fig. 2). SOX2 bound IMPa3 through an extensive and contiguous interface across ARM domains 1-9 of IMPa3 (Fig. 2). The N-terminal NLS (NLS1) was previously reported to be bipartite $7,15,28$, and therefore expected to be bound at both the major and minor sites on IMPa3. However, we found instead that, SOX2 residues Arg40, Lys42, and Arg43 were bound at the minor site (IMPa3 ARM domains 6-8; Fig. 2) and that SOX2 Arg57 was bound at ARM 9, outside of the minor site. The Cterminal NLS (NLS2) was bound in the major site of IMPa3, with SOX2 residues Pro112-Met120 bound to ARM domains 1-4 (Fig. 2). The HMG domain of SOX2, that is located between these NLS regions, formed additional interactions with IMPa3, including SOX2 Lys95 bound to ARM4; SOX2 Arg98 bound to IMPa3 ARM 5; SOX2 His101 and Asp107 bound to ARM 6; and SOX2 Glu104 bound to ARM 7 (see also Supplementary Table 2 for detailed interactions). Overall, this structure showed that, these two distally positioned NLSs within the HMG domain form a contiguous NLS interface on IMPa3 through ARM domains 1-9. That the two NLSs form a single, contiguous binding interface requires a fundamental reevaluation of how SOX proteins are recognized by the nuclear import transport machinery, how mutations in either region can cause disease, and how posttranslational modification can regulate this process ${ }^{29}$. More broadly, the structure provides a striking illustration of how NLS regions in different parts of a molecule can contribute to forming a contiguous NLS on the IMPa adapter.

To investigate the functional importance of the key binding determinants identified in the SOX2:IMPa3 structure, we engineered structure-guided mutations and examined their influence on nuclear localization, stem cell maintenance, and development. A total of 11 single, structure-guided mutations were assessed for their ability to disrupt interaction with IMPa3. These included R40A, K42A, R43A, R56A, R57A, R98A, R113A,
R114A, K115A, T116E (to mimic phosphorylation), and K117A. We found that three of these single-point mutations reduced in vitro binding to IMPa3: K42A, R43A, and K115A (Fig. 3a). Based on a previous study demonstrating that mutating both SOX2 NLS regions impart the most dramatic reduction in nuclear localization ${ }^{30}$ and combining our knowledge of the structural interface, we designed a SOX2 K42A, R43A, and K115A triple mutant (SOX2x3Mut) and tested the effect on the cell biology processes that SOX2 mediates. The SOX2x3Mut binding to IMPa3 was abrogated, as shown in both pull-down (Fig. 3a) and microscale thermophoresis (MST) assays (Fig. 3b).

IMPa3:SOX2 interface mutants affect neural stem cell biology. To examine the impact of these mutations on neural stem cell biology, we compared the ability of SOX 2 and the SOX $2 \times 3$ Mut to maintain cell proliferation in a neural stem cell assay (Fig. 4). Neonatal mouse neural stem/progenitor cells (NSC) can be exponentially expanded for extended periods of time (several months) in vitro; in contrast, Sox2-deleted (Sox2-l-) NSC replicate slowly, and progressively lose their ability to self-renew until the culture becomes completely exhausted ${ }^{31,32}$. We attempted to rescue the ability of Sox $2^{-1-}$ NSC to long-term selfrenew by transducing them with lentiviruses expressing human wild-type SOX2 (ref. ${ }^{32}$ ) or SOX2x3Mut (Fig. 4a). Sox $2^{-1-}$ NSC transduced with wild-type Sox 2 recovered the ability to efficiently self-renew (Fig. 4b, c), growing with kinetics comparable to wildtype NSC (doubling time: $41.81 \pm 7.45 \mathrm{~h}$ for mutant cells transduced with wild-type Sox2, versus $44.22 \pm 4.95 \mathrm{~h}$ for wild-type cells, $n=3$ independent experiments; error is standard deviation). In contrast, NSC transduced with the SOX2x3Mut demonstrated inefficient expansion, progressively slowing until a plateau was reached, after which their numbers started to decline (Fig. 4b, c). In an independent experiment with NSC from a different Sox2 
a

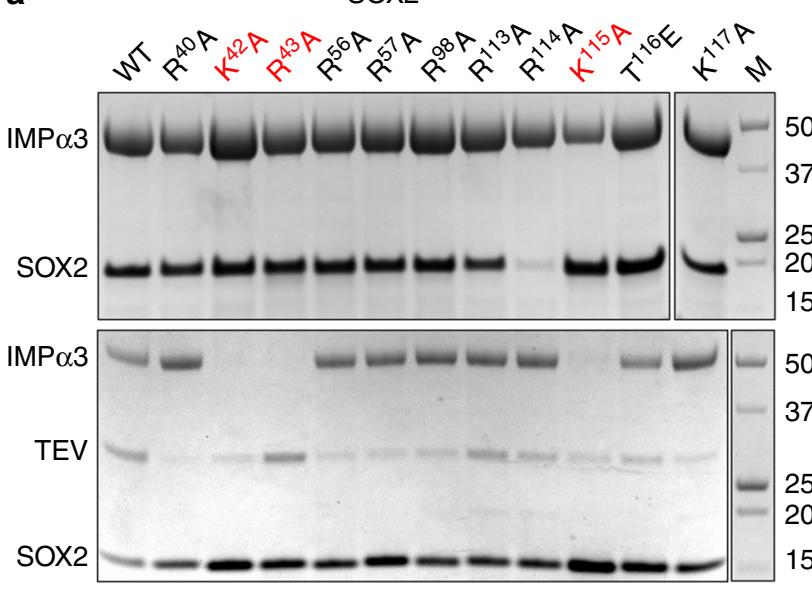

SOX2

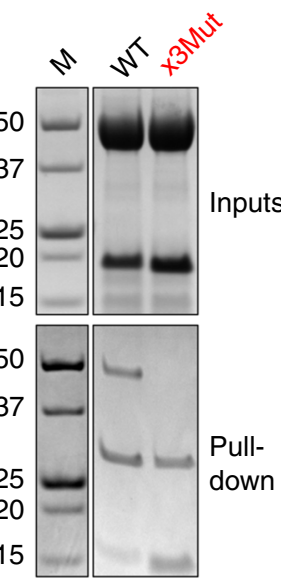

b

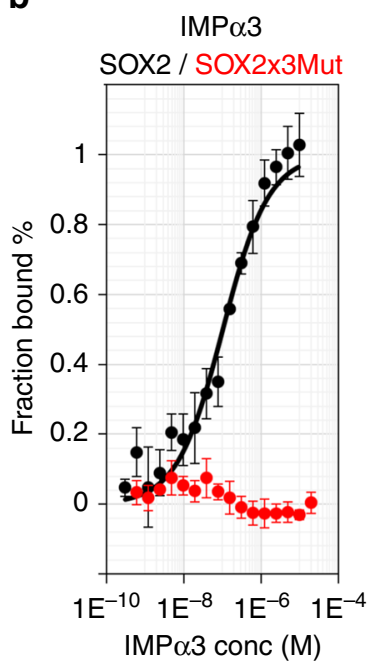

Fig. 3 Structure-guided point mutations in SOX2 disrupt binding to IMPo3. a Mutations within SOX2 were introduced based on the interaction interface and were tested in pull-down assays. His-tagged SOX2 constructs was immobilized on $\mathrm{Ni}^{2+}$ agarose beads in the presence of IMP $\alpha 3$ (see input), washed, and eluted through TEV cleavage. Three single-point mutations disrupted binding: K42A, R43A, and K115A. A single SOX2 triple mutant incorporating three mutations was confirmed to not interact with IMP $\alpha 3$ in the equivalent pull-down experiment. See also Supplementary Fig. 6 for uncropped gels. Results were reproduced independently in three separate experiments with similar results. $\mathbf{b}$ The effect on binding was also assessed in MST assays, where SOX2 was shown to bind with $102 \pm 15 \mathrm{~nm}(n=3$, where $n$ represents three independent experiments) affinity, whereas no binding of SOX2x3Mut (red) could be detected. The data points are presented as mean values \pm standard deviation.

a

d

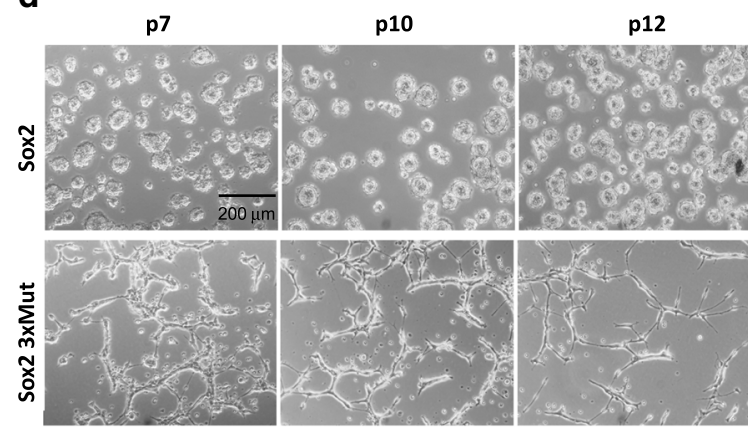

b

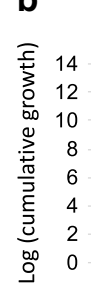

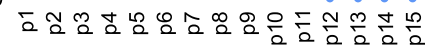
Passages

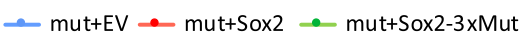

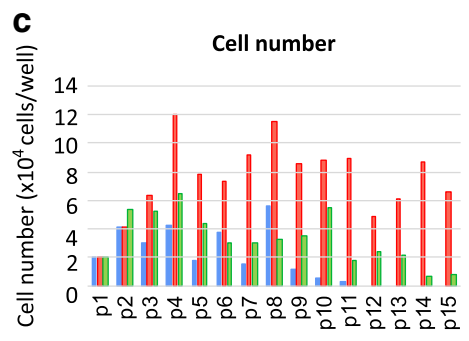

Passages

$\square$ mut+EV $\square$ mut+Sox2 $\square$ mut+Sox2-3xMut

e

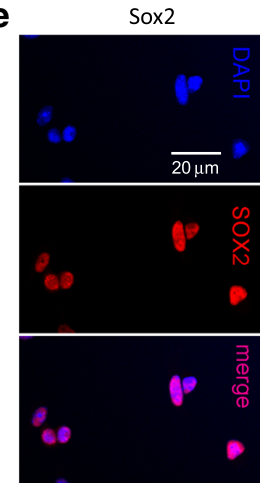

Sox2 3xMut

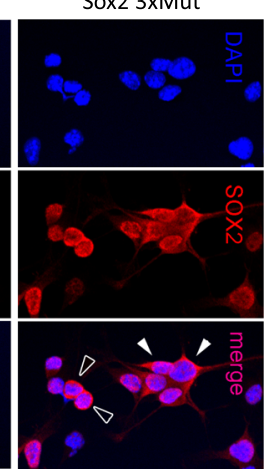

f

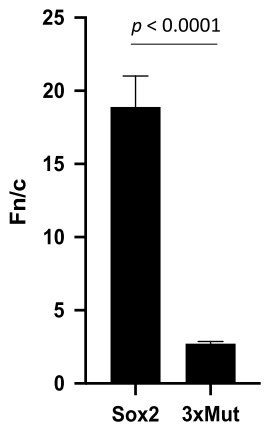

Fig. 4 The SOX2x3Mut protein is severely impaired in its ability to maintain long-term self-renewal of SOX2-deleted NSC. a Schematic representation

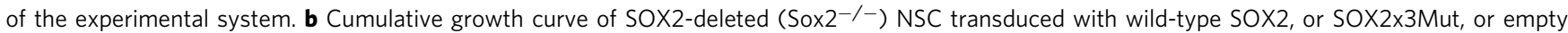
vector (EV). c Number of cells recovered at each passage, after plating 20,000 cells. d Microscopic appearance of SOX2- and SOX2×3Mut-transduced cells, at the indicated passages. Results were reproduced independently in three separate experiments with similar results. e Confocal microscopy of

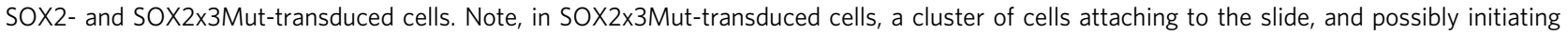
differentiation. $\mathbf{f}$ Digitized images of SOX2 and SOX2×3Mut-transduced cells were quantitated to determine the nuclear ( $\mathrm{Fn}$ ) to cytoplasmic ( $\mathrm{Fc}$ ) florescence ratio $(\mathrm{Fn} / \mathrm{c})$ according to the formula $\mathrm{Fn} / \mathrm{c}=(\mathrm{Fn}-\mathrm{Fb}) /(\mathrm{Fc}-\mathrm{Fb})$ where $\mathrm{Fb}$ is background fluorescence. Results are presented as the mean $\mathrm{Fn} / \mathrm{c} \pm$ SEM ( $n=46$ and 96 independent cells for the SOX2 and SOX2×3Mut samples respectively). $P$ value determined by using a two-tailed unpaired Welch's $T$ test in Graphpad prism 8 software; no adjustments were made for multiple comparisons. The precise $P$ value of 0.0000000011 is displayed as $<0.0001$. See also Supplementary Fig. 7 for data point distribution used to generate mean and SEM values. 
mutant mouse transduced with the same vectors, essentially identical results were obtained, with growth curves closely overlapping those of the first experiment (Supplementary Fig. 1). NSC transduced with SOX2x3Mut had a clear tendency to attach to the plastic, elongate, and aggregate (a possible sign of initial differentiation), in contrast to Sox $2^{-1}$ - NSC transduced with wild-type SOX2, that formed neurospheres, as expected for normal cells (Fig. 4d). FACS analysis of the transduced cells compared to untransduced cells, measuring GFP expressed from the lentiviral vector, showed that in the same culture the transduced cells rapidly exceeded the untransduced cells in number, demonstrating a growth advantage over the rapidly declining SOX2 $2^{-1-}$ NSC. Notably, NSC transduced with SOX $2 \times 3$ Mut also grew quicker and for longer than untransduced SOX2 mutant NSC (or empty-vector (EV)-transduced NSC, Supplementary Fig. 2), suggesting that SOX2x3Mut may retain some activity, though clearly too low to maintain efficient long-term growth (Fig. 4b, c). Confocal microscopy (Fig. 4e, f) demonstrated predominantly cytoplasmic localization in most of the SOX2x3Mut-transduced NSC, whether they had initiated differentiation (white arrowheads) or not (black arrowheads); in contrast, SOX2 was invariably nuclear in wild-type SOX2-transduced NSC. These results indicate a clear correlation between the predominantly cytoplasmic localization of the SOX $2 \times 3$ Mut, and the severe impairment of its ability to sustain long-term NSC selfrenewal.

Homologous SOX2:IMPa3 mutations affect Drosophila development. The role of SOX2 in mammalian embryogenesis is well established, with Sox $2^{-1-}$ known to be embryonic lethal in mice. The key roles of SOX2 are conserved throughout metazoans, with the SOX2 homolog in Drosophila, Dichaete $(87 \%$ sequence identity to human SOX2 in the HMG domain and NLS-binding regions), playing a key role in central nervous system development. To examine if this interface is required for Dichaete function, we generated transgenic strains that expressed HAtagged Dichaete or the orthologous Dichaete3xMut (Dichaete K143A, R144A, and K216 A) from an upstream activation sequence (UAS) promoter. We drove expression using ptc-Gal4 $\left(\mathrm{P}\{\mathrm{GawB}\} \mathrm{ptc}^{559.1}\right)$ that expresses in multiple tissues during development, including third instar salivary glands. Ectopic expression of Dichaete results in developmental defects when expressed from a variety of promoters ${ }^{33-35}$, and we also observed that no ptc-Gal4, UAS-Dichaete adults emerged (0/63 siblings, Supplementary Fig. 3), indicating that it results in lethality when raised at $25^{\circ} \mathrm{C}$. In contrast, expression of Dichaete $3 x M u t$ had no effects upon development and ptc-Gal4, UAS-Dichaete3xMut animals emerged at approximately a Mendelian ratio (37/93 siblings, Supplementary Fig. 3). We were able to isolate third instar larval salivary glands from both allelic combinations and used an anti-HA antibody to observe the intracellular localization of the ectopically expressed Dichaete proteins. Wild-type HA-Dichaete was predominantly localized in nuclei of both the polytene salivary gland cells and the salivary duct cells, whereas HADichaete3xMut localization was much more cytoplasmic. This mislocalization explains why expression of the Dichaete3xMut did not produce a phenotype, as it was not efficiently translocated into the nucleus where it could have an effect upon target gene expression (Fig. 5).

Structural basis for SOX2 specificity toward IMPa isoforms. SOX2 shows specificity toward IMPa isoforms during neural stem cell differentiation ${ }^{14}$. Moreover, many transcription factors and viral proteins exhibit specificity for IMPa isoforms, but the molecular mechanisms are unclear and analysis is complicated because of the high level of conservation of NLS-binding sites across IMPa isoforms. To assess whether SOX2 interacts differentially with IMP $\alpha$ isoforms, we performed an immunoprecipitation assay with representative members of each IMPa subfamily (SF; SF1:a1, SF2:a3, and SF3:a5/7; Fig. 6a). Here, we expressed Flag-tagged IMPa1, $a 3$, and $a 7$ together with HA-tagged SOX2, and performed HA-immunoprecipitation and western analysis. We found that SOX2 bound IMPa3, whereas interaction with IMP 1 1 and IMP 47 was not detected (Fig. 6a). As expected, the SOX $2 \times 3$ Mut demonstrated a loss of interaction with IMPa3. We performed a bead-binding assay using bacterially expressed Histagged SOX2 immobilized on $\mathrm{Ni}^{2+}$ agarose that was able to bind IMPa3 directly, whereas IMPa1, $\alpha 2$, and $\alpha 5$ (same SF as IMPa7) bound more weakly (Fig. 6b). Next, we compared the affinity of these interactions using MST. Here we found that IMPa3 bound SOX2 with the strongest affinity, with a $K_{\mathrm{D}}$ of $102 \pm 15 \mathrm{nM}$ $($ mean $+\mathrm{SD}, n=3$ ), whereas IMPa1, and a5 bound with significantly lower affinity with a $K_{\mathrm{D}}$ of $447 \pm 27 \mathrm{nM}(P<0.0001)$ and $247 \pm 30 \mathrm{nM}(P=0.0017)$, respectively (Fig. 6c). Overall, our results indicate that $\mathrm{SOX} 2$ interacts most strongly with the IMPa3 isoform.

To investigate the possible basis for IMPa isoform specificity, we aligned the amino acid sequences of IMPa isoforms and compared the binding interface residues (Supplementary Fig. 4). We found that of the 28 binding interface residues on IMPa3, 25 are identical across all IMPa isoforms and the remaining 3 are conserved. This suggests that differences in the IMPa NLSbinding sites are not responsible for isoform specificity. To further probe the basis of isoform specificity, we solved the structures of SOX2 bound to both IMPa2 and IMPa5 (2.7 and $2.8 \AA$ resolution respectively). Consistent with the cellular and in vitro binding data, we observed differences at the SOX2:IMPa isoform interfaces, with that from IMPa3 being more extensive. The IMPa3:SOX2 interface was mediated through a buried surface area of $2034 \AA^{2}, 14$ salt bridges, and 34 hydrogen bonds. In comparison, the IMPa2:SOX2 interaction interface buried $1082 \AA^{2}$ of surface area, and contained 4 salt bridges and 24 hydrogen bonds. The IMPa5:SOX2 interface buried $1106 \AA^{2}$ of surface area, and contained 4 salt bridges and 23 hydrogen bonds (see Supplementary Tables 2-4 for detailed interactions). Moreover, superimposing the IMPa isoform structures indicated that local differences in IMPa structure could contribute to the differences in affinity for SOX2 (Fig. 7). Although the overall structures of the IMPa isoforms were very similar (RMSD between IMPa3 with IMPa2 and IMPa5 of 1.5 and $1.9 \AA$, respectively), we found that the ARM 7 domain is positioned differently in IMPa 3 compared to IMPa 2 and IMPa5. This region is where the SOX2 HMG domain interacts with IMPa3, but this interaction was not observed in the IMPa2 and IMPa5 crystals, where instead the HMG domain appeared to be disordered. The position adopted by the ARM 7 domain in both IMPa2 and IMPa5 would generate a steric clash with SOX2 that could impair its binding (Fig. 7). Furthermore, Pro106 of SOX2, that induces a sharp bend adjacent to the SOX-HMG domain, would clash with the main chain of both IMPa 2 and a 5 ARM 7. In contrast, the ARM 7 domain of IMPa3 is set back by $4 \AA$, and so provides a favorable interaction with the HMG domain. In addition, SOX2 cis-Pro44 is also positioned close to the ARM 7 interface. These two prolines lie at either end of the SOX2 HMG domain and facilitate the extended interactions with IMPa3 across ARM domains 1-9 that are not possible with IMPa2 and $\alpha 5$. The reduced binding of the R43A-SOX2 to IMPa 3 was consistent with the position of ARM 7 contributing to the interaction because Arg43 forms the majority of the interactions with this region (Fig. 3). The different positioning of ARM 7 within each isoform was independent of the type of cargo bound (Supplementary 
a

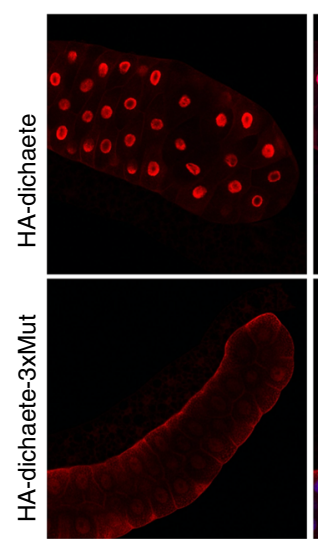

DAPI (nuclei) + HA

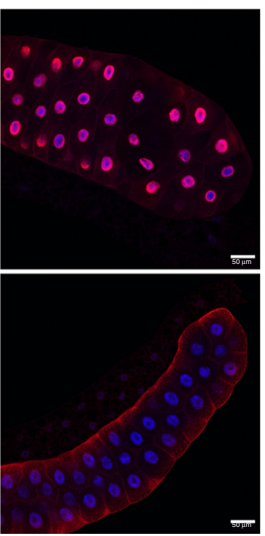

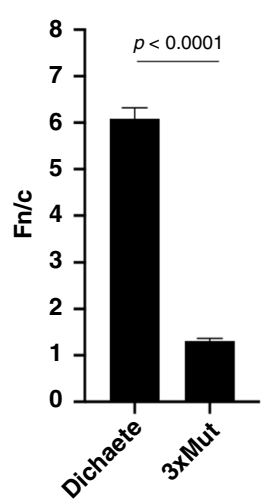

b

Anti HA

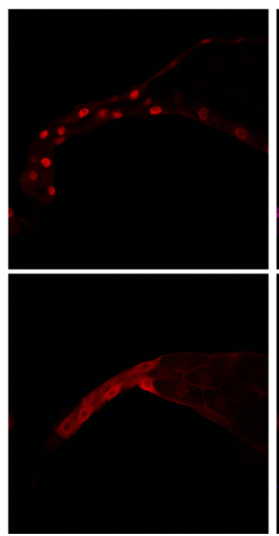

$\mathrm{DAPI}$ (nuclei) + HA

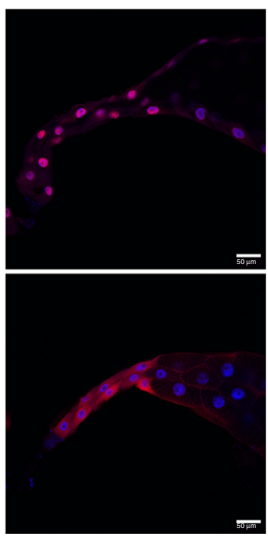

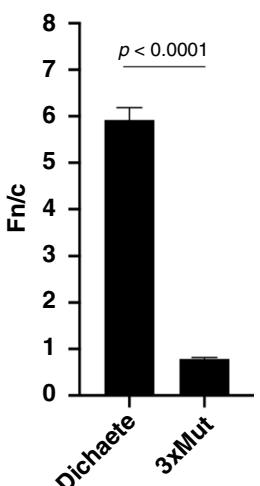

Fig. 5 Loss of the orthologous K42, R43, and K115 residues in Drosophila Dichaete (SOXB 2-1) impairs nuclear localization. a HA-tagged Dichaete exhibits predominately nuclear localization when expressed in salivary gland cells, and $\mathbf{b}$ in salivary duct cells. In contrast, the Dichaete3xMut allele is distributed between nucleus and cytoplasm in salivary gland cells, and is strongly cytoplasmic in salivary duct cells. Scale bar $=50 \mu m$. Digitized images were quantitated to determine the nuclear ( $\mathrm{Fn}$ ) to cytoplasmic (Fc) florescence ratio (Fn/c) as per the legend to Fig. 4, and results are presented as the mean Fn/c \pm SEM ( $n=62$ and 51 (salivary gland), or 52 and 20 (salivary duct) independent cells for the HA-Dichaete and HA-Dichaete3xMut samples respectively). $P$ value determined by using a two-tailed unpaired Welch's $T$ test in Graphpad prism 8 software; no adjustments were made for multiple comparisons. The precise $P$ value could not be generated, but less $<0.000000000000001$ and displayed as $<0.0001$. See also Supplementary Fig. 8 for distribution of data points used to generate mean and SEM values.

Fig. 5), suggesting that this region does not adjust to accommodate different cargo.

\section{Discussion}

SOX proteins localize to the nucleus where they alter nucleosome structure and function ${ }^{36}$, play critical roles in development, and are associated with many cancers. For example, mutations in the SRY protein, within either the N- or C-terminal NLS, reduce the ability of SRY to translocate to the nucleus in sex reversed patients $9,17,18$. In addition, mutations in the NLS region of SOX9 result in reduced nuclear accumulation in campomelic dysplasia patients with XY sex reversal ${ }^{12}$, and SOX10 mutants that fail to localize to the nucleus cause Waardenburg syndrome, resulting in sensorineural hearing defects and auditory-pigmentary disorder $^{37}$. SOX2 plays a critical role in $\mathrm{PSCs}^{3,6,14}$, in neural and other stem cell type maintenance ${ }^{31,32}$, and in developmental and tumor biology $3-6,14,16,38-40$. SOX proteins may either maintain or antagonize tumorigenesis ${ }^{38-42}$. Cytoplasmic SOX9 correlates with poor clinical cancer outcomes, including both shorter diseasespecific survival and relapse-free survival ${ }^{19}$. SOX9 is localized in the cytoplasm of $25-30 \%$ invasive ductal carcinomas and lymph node metastases, and its cytoplasmic accumulation significantly correlates with enhanced proliferation in breast tumors ${ }^{43}$. Cytoplasmic SOX18 correlates with poor patient outcome in adenocarcinoma and is associated with non-small cell lung cancer progression ${ }^{44}$. Moreover, many translational modifications occur within the SOX NLS regions (reviewed in ref. ${ }^{45}$ ). The proposal that SOX proteins localize to the nucleus through two NLSs made it difficult to understand how mutation or modification in only one NLS would affect function because there appeared to be a high level of redundancy provided by the remaining NLS. However, our results demonstrate that these two NLS regions bind as a single continuous interface on IMPa, and so provide a more easily understood mechanistic basis for contextualizing SOX function and how it can be impaired by mutations in the NLSHMG region of the molecule. The structural insights from our study may also assist with contextualizing how mutations in other SOX proteins may cause aberrations in nuclear transport and disease. While there are no naturally occurring mutants in the NLSs of SOX2 that have been documented, SRY mutants have been shown to impede nuclear localization and result in sex reversal. Mutations such as SRY R62 $\mathrm{G}^{15}, \mathrm{R} 75 \mathrm{M}^{46}$, and $\mathrm{R} 76 \mathrm{P}^{47}$, located within the NLS1 (bipartite region) of SOX proteins, were shown to bind within the IMPA minor site (IMPa3 ARMs 6-8) and ARM 9 in this study. Similarly, the NLS2 region harbors mutations, such as SRY R133W ${ }^{48}$, shown to bind at the major site of IMPa3 (within ARM3). It is unlikely however that the interfaces identified in this study can be used to attribute all diseasecausing mutations across the SOX family since these sites are also subject to complex regulation, including calmodulin binding (also shown to regulate nuclear import). This may explain for example why some disease-causing mutations, such as SRY R76P ${ }^{47}$ (equivalent to SOX2 Arg57), shown to be important for nuclear import regulation through calmodulin, did not disrupt the IMPA3:SOX2 interaction ${ }^{8}$.

Establishing the mechanism by which nuclear cargoes are recognized specifically by different IMPa isoforms is critical for understanding many key regulatory, developmental, and cancerrelated processes. For example, neural differentiation of embryonic stem cells is mediated by IMPa isoform switching such that Oct3/4 is driven to the nucleus by IMPa1 in undifferentiated stem cells; however, during neural development, upregulation of IMPa3/5 mediates SOX2/Brn2 nuclear import and neural differentiation ${ }^{14}$. Moreover, SOX proteins may also use alternate pathways for import, such as IMP $\beta^{49}$, calmodulinmediated pathway ${ }^{12}$, or exportin-4 (ref. ${ }^{50}$ ), suggesting that import may be cell or tissue dependent. Structural insights into isoform specificity are limited and our present understanding of cargo binding to different receptor isoforms is limited to RCC1 (ref. ${ }^{24}$ ), influenza A PB2 (ref. ${ }^{51}$ ), and Henipavirus W proteins. It is noteworthy that viral cargo show some of the most remarkable specificity, and early indications suggest this may be an important strategy for viruses, since innate immune responses also require the nuclear transport of STAT1 and NF-kB through isoforms specificity of IMPa3 (ref. ${ }^{52}$ ). Viral accessory proteins have been shown to specifically inhibit transport of these proteins to the nucleus and block innate immune responses. The ability of viruses to specifically target nuclear import to dampen immune responses, while not altering the import of cellular proteins important for cell maintenance (and viral replication), is likely an 
a

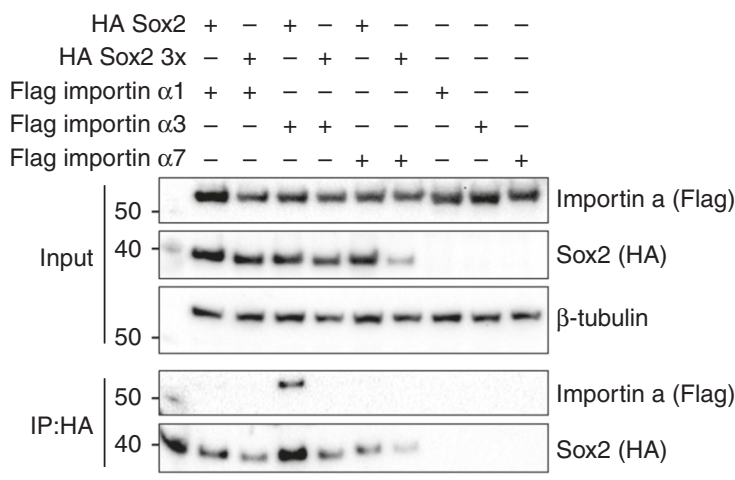

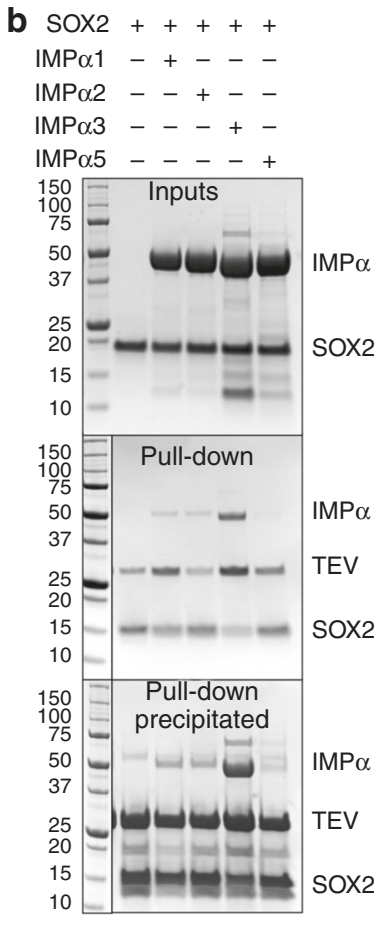

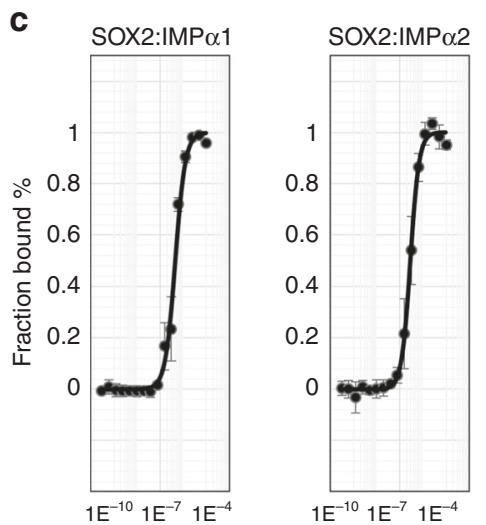

IMP $\alpha$ concentration $[\mathrm{M}]$

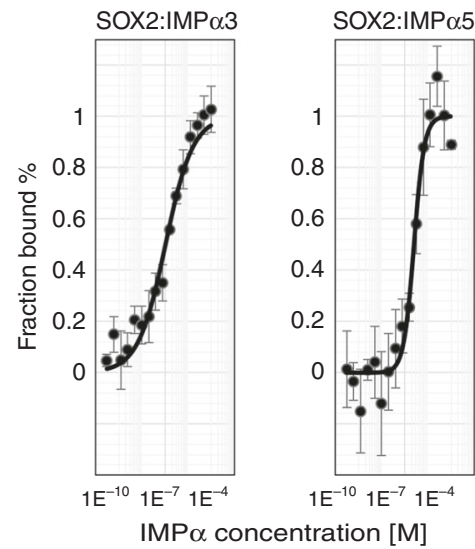

Fig. 6 SOX2 exhibits specificity for IMPo3. a Co-immunoprecipitation assay performed with HA antibody on lysates of HEK293T cells expressing Flagtagged importin from each subfamily (SF; SF1: $\alpha 1, \mathrm{SF} 2: \alpha 3, \mathrm{SF} 3: \alpha 7$ ) and HA-tagged full length SOX2 or SOX3xmut as indicated. A molecular weight marker is present in lane 1. The ColP was repeated three times; a representative western blot is shown. Western blots were performed for HA and Flag. IP immunoprecipitation (see also Supplementary Fig. 9 for uncropped gels). b Pull-down assays using recombinant proteins expressed in E. coli. A representative from each subfamily was expressed. His-tagged SOX2 WT (wild type) was immobilized on $\mathrm{Ni}^{2+}$ agarose beads in the presence of SF1: $\alpha 1$ and $\alpha 2$, SF2: $\alpha 3$, or SF3: $\alpha 5$ as indicated, (see input), washed, and eluted through TEV cleavage. The elution was further concentrated by precipitation to confirm binding differences between IMP $\alpha 3$ and the other isoforms. See also Supplementary Fig. 10 for uncropped gels. Results were reproduced independently in three separate experiments with similar results. c Binding affinities of SOX2 to IMPas within different subfamilies. Sments OX2 bound to IMP $\alpha 1$ with $447 \pm$ $27 \mathrm{~nm}$ affinity $(n=3)$; IMP $\alpha 2$ with $283 \pm 10 \mathrm{~nm}$ affinity $(n=3)$; IMP $\alpha 3$ with $102 \pm 15 \mathrm{~nm}$ affinity $(n=3)$; and IMP $\alpha 5$ with $247 \pm 30 \mathrm{~nm}$ affinity $(n=3)$. In each case, $n=3$ represents three independent experiments. The data points are presented as mean values \pm standard deviation. The differences in binding observed between IMP $\alpha 3$ and other isoforms were significant: IMP $\alpha 1 P=0.000042$; IMP $\alpha 2 P=0.000064$; and IMP $\alpha 5 P=0.0017$. $P$ value determined by using a two-tailed unpaired Welch's $T$ test in Graphpad prism 8 software; no adjustments were made for multiple comparisons.

important viral replication strategy. The greater flexibility of IMPa3 compared with other IMPa isoforms is important for its binding RCC1 selectively ${ }^{24,51}$, whereas we have shown here that the differential positioning of ARM 7 in IMPa3 (the position of which does not change relative to ARM 6 and ARM 8 in all published IMPa3 structures-see Supplementary Fig. 5) makes an important contribution to its selective binding of SOX2, similar to that seen for the W protein of Henipaviruses ${ }^{52}$ (Fig. 8).

Finally, the NLS1 and NLS2 regions within SOX2 that mediate a single interface on IMPa3 are likely to have overlapping functions with SOX2 biology. A recent structure of SOX2 bound to nucleosomes ${ }^{36}$ identified that these regions may adopt strikingly different conformations (Fig. 9 and Supplementary Movie file 1). When bound to IMPa's for nuclear import, these regions are positioned in an open conformation to allow a single continuous interface. In contrast, when bound to nucleosomes, these NLS regions are in close proximity and in a closed conformation. That the IMPa and nucleosome binding sites are overlapping suggests a possible release (and recycling) mechanism for IMPa; however, this requires further experimental investigation.

\section{Methods}

\section{Protein expression}

Plasmids for recombinant protein expression. The SOX2 HMG domain (encoding residues 39-127; UniProtKB P48431), and IMPa lacking the IMP $\beta$-binding domain: IMPa1 (encoding residues 70-529; UniProtKB P52292), IMPa2 (encoding residues 70-529; UniProtKB P52293), IMPa3 (encoding residues 64-521; UniProtKB O00629), IMPa5 (encoding residues 73-538; UniProtKB p52294), all containing an N-terminal TEV cleavage site (ENLYFQS) were codon optimized for expression in Escherichia coli and synthesized by Genscript (Piscataway, NJ). The constructs were cloned into a single BamH1 site within the pET30a + vector.

Recombinant expression and purification. Plasmids were transformed into BL21 (DE3) pLysS cells and expressed, as described in similar studies ${ }^{53,54}$, and using the following procedures. Starter cultures were inoculated into $2 \mathrm{~L}$ baffled flasks containing $500 \mathrm{~mL}$ of expression media consisting of $1 \%(\mathrm{w} / \mathrm{v})$ tryptone, $0.5 \%(\mathrm{w} / \mathrm{v})$ yeast extract, $0.5 \%$ glycerol, $0.05 \%$ glucose, $0.2 \%(\mathrm{w} / \mathrm{v})$ a-lactose, $0.025 \mathrm{M} \mathrm{NH}_{4} \mathrm{SO}_{4}$, $0.05 \mathrm{M} \mathrm{KH}_{2} \mathrm{PO} 4,0.05 \mathrm{M} \mathrm{Na}_{2} \mathrm{HPO} 4,1 \mathrm{mM}$ magnesium chloride, and $50 \mu \mathrm{g} / \mathrm{mL}$ kanamycin. Cells were harvested via centrifugation at $6500 \times g$ and $18^{\circ} \mathrm{C}$ for 30 min and resuspended in phosphate buffer (PB; $50 \mathrm{mM}$ phosphate $\mathrm{pH} 8.0,300 \mathrm{mM}$ $\mathrm{NaCl}$, and $20 \mathrm{mM}$ imidazole). Cells were lysed using two freeze-thaw cycles, and addition of $20 \mathrm{mg}$ lysozyme and $0.5 \mathrm{mg}$ DNaseI.

Purification of 6xHis-tagged proteins were performed by injecting clarified cell lysate onto a GE HisTrap $5 \mathrm{~mL}$ column using PB, washing the column with 15 column volumes, and then eluting over 5 column volumes, using a gradient elution 

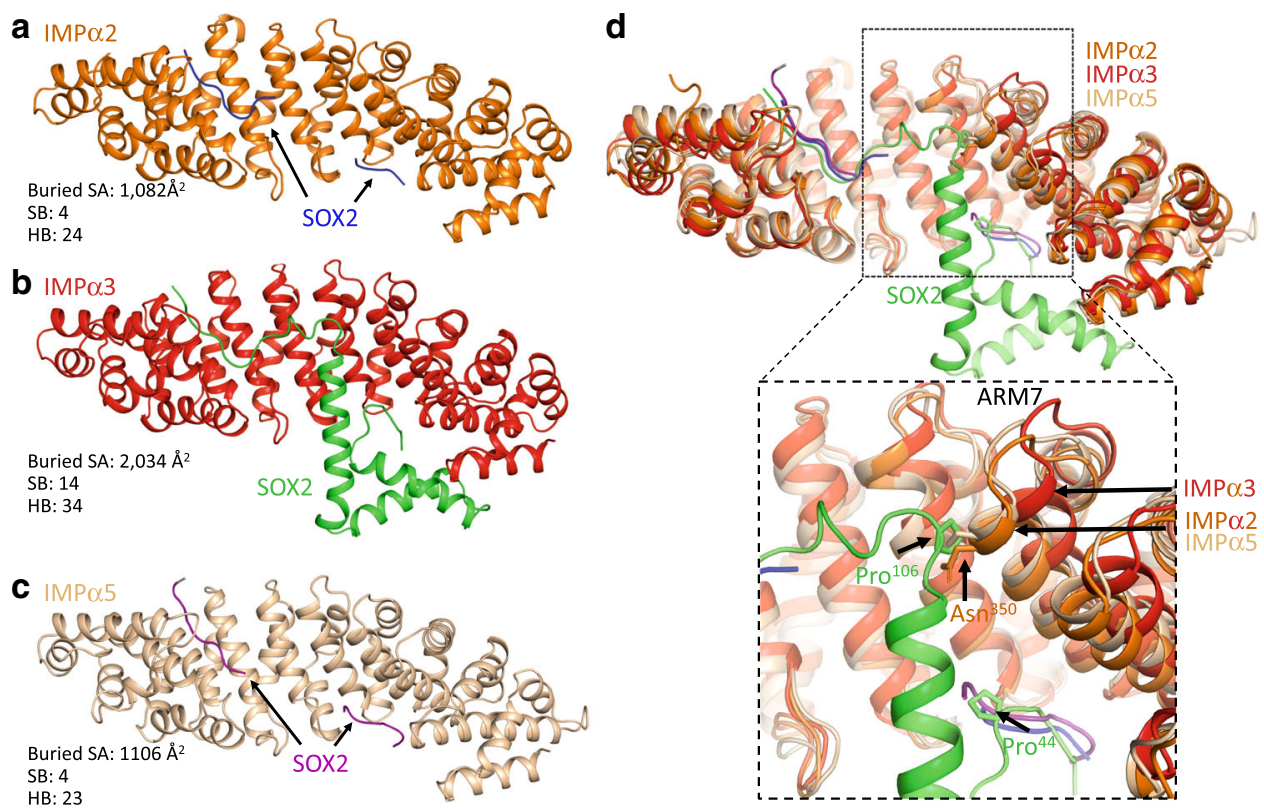

Fig. 7 Structures of SOX2 bound to IMP $\alpha$ isoforms from each of the three subfamilies show significant differences in the binding interface. a IMP $\alpha 2$ : SOX2 structure shown with IMP $\alpha 2$ (orange) and SOX2 in blue. The interface was mediated through $1082 \AA^{2}$ of buried surface area, 4 salt bridges, and 24 hydrogen bonds. b The IMP $\alpha 3:$ SOX2 structure exhibits a greater interface, with IMP $\alpha 3$ (red) and SOX2 in (green) burying $2034 \AA^{2}$ of surface area, and mediated by 14 salt bridges, and 34 hydrogen bonds. c The IMP $\alpha 5$ :SOX2 is depicted with IMP $\alpha 5$ in light brown and SOX 2 in purple, buries $1106 \AA^{2}$, and was mediated by 4 salt bridges and 23 hydrogen bonds. $\mathbf{d}$ Superposition of the three structures highlights the positioning of ARM 7 in IMP $\alpha 3$ is conducive to binding the HMG domain, while IMP $\alpha 2$ and IMP $\alpha 5$ are both positioned in a conformation that prevents these interactions.
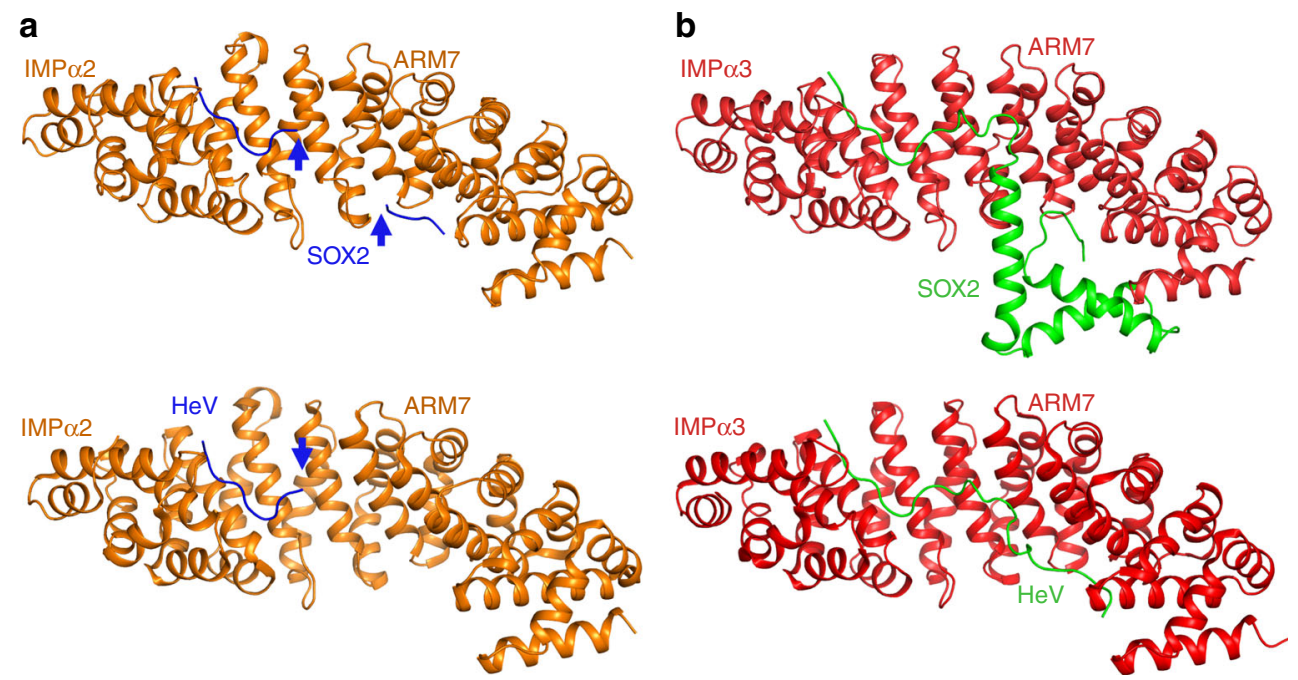

Fig. 8 The positioning of ARM domain $\mathbf{7}$ is also important for specificity of the Hendra virus W protein. a Structure of IMP $\alpha 2$ (shown in light brown, cartoon/ribbon format) bound to SOX2 or Hendra virus W protein ( $\mathrm{HeV}$; shown in blue, cartoon/ribbon format). b Structural overview of SOX2 and HeV (shown in green, cartoon/ribbon format) bound to IMP $\alpha 3$ (shown in red, cartoon/ribbon format). The positioning of the ARM 7 was shown to be important for isoform specificity between IMP $\alpha 2$ and IMP $\alpha 3$.

with high imidazole $(500 \mathrm{mM}$ imidazole, $300 \mathrm{mM} \mathrm{NaCl}$, and $50 \mathrm{mM}$ phosphate $\mathrm{pH}$ 8.0). Samples were pooled, and the affinity tag removed by TEV proteolysis. Sizeexclusion purification of pooled samples was performed on a Superdex $200 \mathrm{pg}$ 26/600 column, using TBS pH 8.0. Eluted proteins were pooled and concentrated using $10 \mathrm{kDa}$ MW centrifuge filters. Complex formation was performed by treating the SOX2 samples with RNAse, and mixing IMPa isoforms with SOX2 in a 1:2 molar ratio. The samples were repurified using size-exclusion chromatography, concentrated using $10 \mathrm{kDa}$ MW centrifuge filters, and aliquoted.

Crystallization, data collection, and processing. All crystals were obtained using the hanging drop vapor diffusion method over a $300 \mu \mathrm{L}$ reservoir solution. IMPa2: SOX2 was crystallized in $750 \mathrm{mM}$ sodium citrate ( $\mathrm{pH} 7.0$ ) and $10 \mathrm{mM}$ DTT, single rod-shaped crystals forming within 14 days. IMPa3:SOX2 crystallized in $1.2 \mathrm{M}$
$\left(\mathrm{NH}_{4}\right)_{2} \mathrm{SO}_{4}, 0.1 \mathrm{M}$ HEPES $\mathrm{pH}$ 6.5, with plate-shaped crystals forming within 3 days. IMPa5:SOX2 crystallized in $0.2 \mathrm{M}$ sodium/potassium phosphate, $20 \%(\mathrm{w} / \mathrm{v})$ PEG3350 with needle-shaped crystals forming within 7 days. X-ray diffraction data were collected at the Australian Synchrotron on the MX1 and MX2 macromolecular beam lines, using an ASDC Quantum 210r, ASDC Quantum 315r detector, and Eiger $16 \mathrm{M}$ detector, respectively ${ }^{55,56}$. Data reduction and integration was performed using iMosflm ${ }^{57}$. Merging, space group assignment, scaling and selection of $5 \%$ reflections for $R_{\text {free }}$ calculations were performed, using Aimless 58 and the CCP4 suite ${ }^{59}$. The anisotropy of the SOX2:IMPA3 and SOX2:IMPA5 data sets was addressed, using the UCLA MBI server (https://services.mbi.ucla.edu/ anisoscale/). Phasing was performed using molecular replacement in Phaser MR, with PDBID 3UL1 (ref. ${ }^{60}$ ) used as a search model for IMPa2:SOX2, PDBID 6BW9 (ref. ${ }^{52}$ ) for IMPa3:SOX2, PDBID 4B18 (ref. ${ }^{61}$ ) for IMPa5:SOX2. Models were built and refined using iterative cycles of $\operatorname{coot}^{62}$ and maximum likelihood phenix 


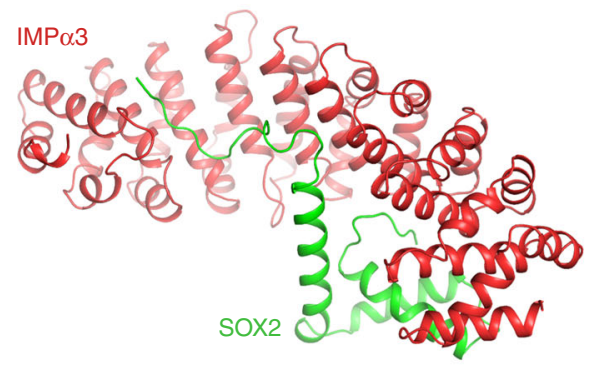

b

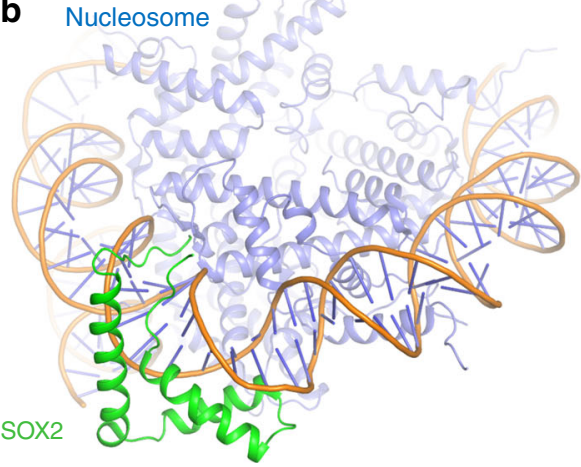

C

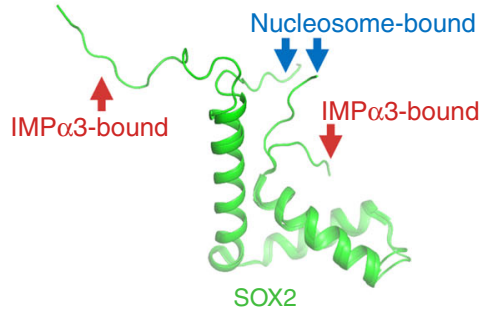

Fig. 9 SOX2 NLS regions adopt different conformations when bound to importins or nucleosomes. a To gain access to the nucleus, SOX2 binds IMP $\alpha 3$ with the NLS regions in an extended conformation to form a single interface. $\mathbf{b}$ When localized to the nucleus, these regions adopt a closed conformation for optimal DNA binding ${ }^{36}$. c The regions are mutually exclusive and may suggest a possible release mechanism.

refine ${ }^{63}$. The final models have been validated and deposited to the PDB with accession numbers detailed in Supplementary Table 1 .

Microscale thermophoresis. Binding affinity measurements were performed on a Monolith NT.115 (NanoTemper Technologies). Purified importins were buffer exchanged in $20 \mathrm{mM}$ HEPES, $150 \mathrm{mM} \mathrm{NaCl}, \mathrm{pH} 8.0$, and labeled, using the Monolith his-tag labeling kit (RED-tris-NTA second generation), Nano-Temper labeling kit according to the manufacturer's instructions. Each reaction consisted of $10 \mu \mathrm{L}$ of the labeled SOX2 protein at $50 \mathrm{nM}$, mixed with unlabeled importins at the indicated concentrations. All experiments were measured at $25^{\circ} \mathrm{C}$ with laser off/ on/off times of 5/30/5 s. Experiments were conducted at 20\% light-emitting diode power and 20-40\% MST infrared laser power. Data from three independently performed experiments were fitted to the single binding model via the NT. Analysis software version 1.5.41 (NanoTemper Technologies) using the signal from thermophoresis + T-Jump.

Bead-binding assays. Each assay contained $10 \mu \mathrm{M}$ of SOX2 or SOX2 mutant immobilized on $50 \mu \mathrm{L}$ of Ni-NTA His-bind resin (Cat\#70666), and $20 \mu \mathrm{M}$ of IMPa in a reaction volume of $300 \mu \mathrm{L}$. The resin was washed three times with PB $(50 \mathrm{mM}$ $\mathrm{PB} \mathrm{pH} 8.0,300 \mathrm{mM} \mathrm{NaCl}$, and $20 \mathrm{mM}$ imidazole). After the final wash, $95 \mu \mathrm{L}$ of $\mathrm{PB}$ buffer and $5 \mu \mathrm{L}$ of TEV protease $(5 \mathrm{mg} / \mathrm{mL})$ were added into each reaction, and incubated at RT for $30 \mathrm{~min}$. A total of $30 \mu \mathrm{L}$ of supernatant was removed and analyzed by SDS-PAGE. Where precipitation was used to concentrate the sample, $70 \mu \mathrm{l}$ of the supernatant was mixed with $1 \mathrm{~mL}$ of $100 \%$ ethyl alcohol and incubated overnight at $-20^{\circ} \mathrm{C}$. Samples were then centrifuged and the pellet dissolved in SDS-PAGE loading dye, and analyzed by SDS-PAGE.

Primary ex vivo neural stem/progenitor cell cultures. Brain-derived NSC cultures were obtained from dissected telencephalon of two SOX2-deleted mice at postnatal day $0(\mathrm{P} 0)^{32}$, and grown in fresh medium (FM): Dulbecco's minimal essential medium (DMEM)-F12 with Glutamax (GIBCO), supplemented with 1/50 (vol/vol) B27 (Life Technologies), 1\% of penicillin-streptomycin (Euroclone) supplemented with EGF (10 ng/mL, Tebu-bio) as mitogen (see also refs. ${ }^{31,32,64}$ ).

Lentiviral preparations. Lentiviral vectors were produced by calcium phosphate transfection into the packaging human embryonic kidney cell line $293 \mathrm{~T}$, of the VSV-G plasmid (encoding ENV), CMV R8.74 (packaging), and pRSV-REV (encoding reverse transcriptase) ${ }^{31,65}$. Briefly, after transfection, following replacement with DMEM high glucose (Euroclone), containing 10\% fetal bovine serum (FBS; Sigma), 1\% penicillin-streptomycin (Euroclone), 1\% of L-glutamine (Euroclone), the cells were further incubated for $48 \mathrm{~h}$, and the cell supernatants were collected. Lentiviral vectors were titrated on HEK293T cells by measuring the percentage of eGFP-positive cells by flow cytometry ${ }^{65}$.

\section{NSC transduction}

NSC transduction ${ }^{31}$. SOX2-deleted neurospheres were grown for two passages (3-4 days each) in FM supplemented with bFGF (10 ng/mL, Tebu-bio) and EGF ( $10 \mathrm{ng} / \mathrm{mL}$, Tebu-bio), and for three more passages in FM supplemented with EGF only. For passaging, neurospheres were first incubated in $0.25 \%$ trypsin (GIBCO) for $5 \mathrm{~min}$ at $37^{\circ} \mathrm{C}$ and, subsequently, ovomucoid (Leibovitz's L15 medium (GIBCO) containing trypsin inhibitor (Sigma), bovine serum albumin (BSA; Sigma), and $40 \mu \mathrm{g} / \mathrm{mL}$ DNaseI (Sigma) for $5 \mathrm{~min}$ at $37^{\circ} \mathrm{C}$ ). Neurospheres were then carefully dissociated mechanically by gently pipetting up and down, centrifuged at $265 \times g$ for 4 min and cells were resuspended in $1 \mathrm{~mL}$ of FM, counted, and seeded at a density of $5 \times 10^{5}$ cells/T25 $\mathrm{cm}^{2}$ flask $/ 5 \mathrm{~mL}$, in FM with EGF as mitogen. After $4 \mathrm{~h}$, SOX2-deleted NSCs were transduced with a GFP-SOX2-expressing lentivirus 32,66 , or with the same vector (EV) expressing GFP only (for control) or carrying SOX2x3Mut, at a multiplicity of infection of 8 for each vector, individually. Cells were incubated overnight at $37^{\circ} \mathrm{C}$. The virus was removed by medium change at $24 \mathrm{~h}$ : cells were centrifuged at $180 \times g$ for $4 \mathrm{~min}$ and resuspended in FM with EGF. At every passage, every 3-4 days, cells were dissociated to single cells as above, counted, and replated in FM with EGF at a density of 20,000 cells/well/1 mL, to generate a cumulative growth curve. At every passage, aliquots of transduced cells $(50,000-100,000$ cells, from pooled wells) were fixed using $2 \%$ paraformaldehyde (PFA) and analyzed for GFP fluorescence by CytoFLEX (BeckmanCoulter) to determine the percentage of transduced cells: 10,000 events were analyzed for each sample.

Mice homozygous for a "floxed" Sox2 allele were crossed with mice compound heterozygotes for a $\beta$ geo gene knocked into the Sox2 gene (generating a null Sox2 mutation), and a nestin-cre transgene (which deletes the floxed Sox 2 allele specifically in the nervous system), to obtain mutant mice with homozygous Sox2 deletion in the brain (Favaro et al. ${ }^{32}$ ). Control Sox2-wild-type mice are generated in the same crosses when the nestin-cre gene is not inherired, and have a Sox 2 floxed allele together with an intact Sox 2 gene. Mice were sacrificed at P0 to obtain forebrains for NSC cultures (sex was indifferent). The lines (Favaro et al. ${ }^{32}$ ) are maintained by matings between cousins, and outbred every two to three generations with $\mathrm{B} 6 \mathrm{D} 2 \mathrm{~F} 1$ mice, to maintain the mutant alleles. Mice were housed at a temperature of $19-23^{\circ} \mathrm{C}$, with $40-60 \%$ humidity, and a $13 \mathrm{~h}$ light $/ 11 \mathrm{~h}$ dark cycle. The experiments were approved by the Italian Ministry of Health as conforming to the relevant regulatory standards.

Immunocytochemistry. Transduced SOX2-deleted NSCs were dissociated to single cells and seeded on Matrigel ${ }^{\mathrm{m} w}$-coated glass coverslips at a density of 80,000 cell/ coverslip. After $4 \mathrm{~h}$, cells were fixed for $20 \mathrm{~min}$ with $4 \%$ PFA in phosphate-buffered saline (PBS; pH 7.4) and rinsed three times with PBS. Coverslips were then incubated for $90 \mathrm{~min}$ in PBS containing 10\% normal goat serum, $0.2 \%$ Triton-X100 at room temperature. Coverslips were then incubated with the primary anti-SOX2 antibody (mouse monoclonal IgG2a, 1:100, R\&D Systems), overnight at $4{ }^{\circ} \mathrm{C}$. After thorough washing with PBS, cells were incubated for $45 \mathrm{~min}$ at room temperature with secondary goat anti-mouse IgG2a cross-adsorbed secondary antibody, Alexa Fluor 594, (Thermo Fisher Scientific, Catalog\# A-21135, 1:1000). Coverslips were rinsed three times in PBS and mounted on glass slides with Fluoromount (Sigma) with DAPI (4',6-diamidino-2-phenylindole).

Drosophila expression studies. The Drosophila melanogaster Dichaete coding sequence as cloned into pUASTattB with modification of the stop codon to introduce Gly-Ser residues followed by a 3xHA tag. The Dichaetex3Mut transgene was generated by mutating K42, R43, and K115 to alanine residues (Genscript). Both transgenes were introduced into the same genomic position in the Drosophila genome (BestGene, Inc.) and expression was induced at $25^{\circ} \mathrm{C}$, using the ptc-Gal 4 ( $\mathrm{P}\{\mathrm{GawB}\} \mathrm{ptc}^{559.1}$ ) driver (Bloomington Drosophila Stock Center). Immunostaining was conducted as described previously ${ }^{67}$, and involved salivary glands dissected from wandering third instar larvae in PBS and fixed in $4 \%$ formaldehyde/PBS for $15 \mathrm{~min}$. Following fixation, tissues were washed three times in PBT for $5 \mathrm{~min}$ each (1\% Triton-X100/PBS) and blocked in PBTH (PBT $+5 \%$ horse serum) for $1 \mathrm{~h}$. This was followed by incubation in primary antibody, rat anti-HA 1:100 (SigmaAldrich, cat\#11867423001) overnight at $4{ }^{\circ} \mathrm{C}$. Subsequent to primary antibody incubation, tissues were washed three times in PBT and incubated in secondary antibody, AlexaFluor594 anti-rat 1:500 (ThermoFisher, cat\# A-21209) for $2 \mathrm{~h}$. Tissues were again washed three times in PBT prior to mounting in ProLong Gold Antifade Mountant with DAPI (ThermoFisher, cat\# P36935). Unless otherwise stated, all steps were carried out at room temperature. Samples were then imaged on a Zeiss LSM800 Airyscan confocal microscope. 


\section{Co-immunoprecipitation assay}

Cells. HEK293T cells (CRL-3216) were obtained from ATCC and were maintained in DMEM supplemented with $10 \% \mathrm{FBS}$ and cultured at $37^{\circ} \mathrm{C}$ and $5 \% \mathrm{CO}_{2}$.

Plasmids. Impa1, Impa3, and Impa7 were cloned by PCR into Flag-tagged pCAGGS ${ }^{52}$. pDNR hSOX2 was purchased from Addgene (plasmid \#49389; RRID: Addgene_49389) and cloned by PCR into HA-tagged pCAGGS. The HA-tagged pCAGGS SOX2x3Mut was generated by overlapping PCR.

Co-immunoprecipitation assay. Flag-tagged Impa1, Impa3, and Impa7 $(3 \mu \mathrm{g})$, and HA-tagged SOX2 and SOX2x3Mut $(3 \mu \mathrm{g})$ were transfected into HEK293T cells $\left(1 \times 10^{6}\right)$, using Lipofectamine 2000 (Thermo Fisher Scientific). At $24 \mathrm{~h}$ post transfection, cells were lysed in NP-40 lysis buffer $(50 \mathrm{mM}$ Tris pH 7.5, $280 \mathrm{mM}$ $\mathrm{NaCl}, 0.5 \% \mathrm{NP}-40,0.2 \mathrm{mM}$ EDTA, $2 \mathrm{mM}$ EGTA, 10\% glycerol, and protease inhibitor (complete; Roche, Indianapolis, IN)). EZview Red anti-HA agarose affinity gel beads (Sigma-Aldrich) were preincubated in 3\% BSA in NP-40 lysis buffer for $1 \mathrm{~h}$ at $4{ }^{\circ} \mathrm{C}$. Beads were then incubated with lysates for $30 \mathrm{~min}$ at $4{ }^{\circ} \mathrm{C}$, washed ten times in NP-40 lysis buffer, and eluted using HA peptide (Sigma-Aldrich) at $4{ }^{\circ} \mathrm{C}$ for $30 \mathrm{~min}$. Whole cell lysates and co-precipitation samples were run on $10 \%$ Bis-Tris Plus polyacrylamide gels (ThermoFisher) and transferred to PVDF membrane (Bio-Rad). Membranes were probed with rabbit anti-HA (Invitrogen cat\# 71-5500, 1:3000), rabbit anti-Flag (Sigma-Aldrich cat\# F7425, 1:3000), mouse anti- $\beta$-tubulin (Sigma-Aldrich cat\# T8328, 1:10,000), and anti-rabbit IgG HRPlinked antibody (Cell Signaling cat\# 7074 S, 1:10,000) or anti-mouse IgG HRPconjugate antibody (EMD Millipore cat\# AP308P, 1:10,000), and were developed by Western Lightening Plus ECL (Perkin Elmer) and imaged on a ChemiDoc MP Imaging System (Bio-Rad).

Reporting summary. Further information on research design is available in the Nature Research Reporting Summary linked to this article.

\section{Data availability}

The data that support this work are available from the corresponding author upon reasonable request. Protein Data Bank files associated with the structures generated in this study have been deposited to the Protein Data Bank, and issued PDB accession codes 6WX7, 6WX8, and 6WX9.

Received: 6 July 2020; Accepted: 26 October 2020; Published online: 04 January 2021

\section{References}

1. Grimm, D. et al. The role of SOX family members in solid tumours and metastasis. Semin. Cancer Biol. 67, 122-153 (2019).

2. She, Z. Y. \& Yang, W. X. SOX family transcription factors involved in diverse cellular events during development. Eur. J. Cell Biol. 94, 547-563 (2015).

3. Avilion, A. A. et al. Multipotent cell lineages in early mouse development depend on SOX2 function. Genes Dev. 17, 126-140 (2003).

4. Papanayotou, C. et al. A mechanism regulating the onset of Sox2 expression in the embryonic neural plate. PLoS Biol. 6, e2 (2008).

5. Donner, A. L., Episkopou, V. \& Maas, R. L. Sox2 and Pou2f1 interact to control lens and olfactory placode development. Dev. Biol. 303, 784-799 (2007).

6. Masui, S. et al. Pluripotency governed by Sox 2 via regulation of Oct $3 / 4$ expression in mouse embryonic stem cells. Nat. Cell Biol. 9, 625-635 (2007).

7. Kaur, G. et al. Calmodulin-dependent nuclear import of HMG-box family nuclear factors: importance of the role of SRY in sex reversal. Biochem. J. 430, 39-48 (2010).

8. Sim, H. et al. Defective calmodulin-mediated nuclear transport of the sexdetermining region of the $\mathrm{Y}$ chromosome (SRY) in XY sex reversal. Mol. Endocrinol. 19, 1884-1892 (2005).

9. Li, B. et al. Human sex reversal due to impaired nuclear localization of SRY. A clinical correlation. J. Biol. Chem. 276, 46480-46484 (2001).

10. Poulat, F. et al. Nuclear localization of the testis determining gene product SRY. J. Cell Biol. 128, 737-748 (1995).

11. She, Z. Y. \& Yang, W. X. Nucleocytoplasmic shuttling of SOX14A and SOX14B transcription factors. Oncotarget 8, 46955-46968 (2017).

12. Argentaro, A. et al. A SOX9 defect of calmodulin-dependent nuclear import in campomelic dysplasia/autosomal sex reversal. J. Biol. Chem. 278, 33839-33847 (2003).

13. Rehberg, S. et al. Sox10 is an active nucleocytoplasmic shuttle protein, and shuttling is crucial for Sox10-mediated transactivation. Mol. Cell Biol. 22, 5826-5834 (2002).

14. Yasuhara, N. et al. Triggering neural differentiation of ES cells by subtype switching of importin-alpha. Nat. Cell Biol. 9, 72-79 (2007).
15. Sudbeck, P. \& Scherer, G. Two independent nuclear localization signals are present in the DNA-binding high-mobility group domains of SRY and SOX9. J. Biol. Chem. 272, 27848-27852 (1997).

16. Andreu-Agullo, C., Maurin, T., Thompson, C. B. \& Lai, E. C. Ars2 maintains neural stem-cell identity through direct transcriptional activation of Sox2. Nature 481, 195-198 (2011).

17. Chen, Y. S., Racca, J. D., Phillips, N. B. \& Weiss, M. A. Inherited human sex reversal due to impaired nucleocytoplasmic trafficking of SRY defines a male transcriptional threshold. Proc. Natl Acad. Sci. USA 110, E3567-E3576 (2013).

18. Harley, V. R. et al. Defective importin beta recognition and nuclear import of the sex-determining factor SRY are associated with XY sex-reversing mutations. Proc. Natl Acad. Sci. USA 100, 7045-7050 (2003).

19. Sumita, Y. et al. Cytoplasmic expression of SOX 9 as a poor prognostic factor for oral squamous cell carcinoma. Oncol. Rep. 40, 2487-2496 (2018).

20. Goldfarb, D. S., Corbett, A. H., Mason, D. A., Harreman, M. T. \& Adam, S. A. Importin alpha: a multipurpose nuclear-transport receptor. Trends Cell Biol. 14, 505-514 (2004).

21. Cingolani, G., Petosa, C., Weis, K. \& Muller, C. W. Structure of importin-beta bound to the IBB domain of importin-alpha. Nature 399, 221-229 (1999).

22. Milles, S. et al. Plasticity of an ultrafast interaction between nucleoporins and nuclear transport receptors. Cell 163, 734-745 (2015).

23. Pumroy, R. A. \& Cingolani, G. Diversification of importin-alpha isoforms in cellular trafficking and disease states. Biochem. J. 466, 13-28 (2015).

24. Sankhala, R. S. et al. Three-dimensional context rather than NLS amino acid sequence determines importin alpha subtype specificity for RCC1. Nat. Commun. 8, 979 (2017).

25. Ao, Z. et al. Importin alpha3 interacts with HIV-1 integrase and contributes to HIV-1 nuclear import and replication. J. Virol. 84, 8650-8663 (2010).

26. Reid, S. P. et al. Ebola virus VP24 binds karyopherin alphal and blocks STAT1 nuclear accumulation. J. Virol. 80, 5156-5167 (2006).

27. $\mathrm{Xu}, \mathrm{W}$. et al. Ebola virus VP24 targets a unique NLS binding site on karyopherin alpha 5 to selectively compete with nuclear import of phosphorylated STAT1. Cell Host Microbe 16, 187-200 (2014)

28. Malki, S., Boizet-Bonhoure, B. \& Poulat, F. Shuttling of SOX proteins. Int. J. Biochem. Cell Biol. 42, 411-416 (2010).

29. Kamachi, Y. \& Kondoh, H. Sox proteins: regulators of cell fate specification and differentiation. Development 140, 4129-4144 (2013).

30. Li, J. et al. A dominant-negative form of mouse SOX2 induces trophectoderm differentiation and progressive polyploidy in mouse embryonic stem cells. $J$. Biol. Chem. 282, 19481-19492 (2007).

31. Bertolini, J. A. et al. Mapping the global chromatin connectivity network for Sox2 function in neural stem cell maintenance. Cell Stem Cell 24, 462-476 e466 (2019).

32. Favaro, R. et al. Hippocampal development and neural stem cell maintenance require Sox2-dependent regulation of Shh. Nat. Neurosci. 12, 1248-1256 (2009).

33. Ma, Y. et al. Functional interactions between Drosophila bHLH/PAS, Sox, and POU transcription factors regulate CNS midline expression of the slit gene. J. Neurosci. 20, 4596-4605 (2000).

34. Mukherjee, A., Shan, X., Mutsuddi, M., Ma, Y. \& Nambu, J. R. The Drosophila sox gene, fish-hook, is required for postembryonic development. Dev. Biol. 217, 91-106 (2000).

35. Russell, S. The Drosophila dominant wing mutation Dichaete results from ectopic expression of a Sox-domain gene. Mol. Gen. Genet. 263, 690-701 (2000).

36. Dodonova, S. O., Zhu, F., Dienemann, C., Taipale, J. \& Cramer, P. Nucleosome-bound SOX2 and SOX11 structures elucidate pioneer factor function. Nature 580, 669-672 (2020).

37. Wang, X. P. et al. Functional analysis of a SOX10 gene mutation associated with Waardenburg syndrome II. Biochem. Biophys. Res. Commun. 493, 258-262 (2017).

38. Li, Y. et al. Overexpression of SOX2 is involved in paclitaxel resistance of ovarian cancer via the PI3K/Akt pathway. Tumour Biol. 36, 9823-9828 (2015).

39. Piva, M. et al. Sox 2 promotes tamoxifen resistance in breast cancer cells. EMBO Mol. Med. 6, 66-79 (2014).

40. Schaefer, T. et al. Molecular and functional interactions between AKT and SOX2 in breast carcinoma. Oncotarget 6, 43540-43556 (2015).

41. Favaro, R. et al. Sox2 is required to maintain cancer stem cells in a mouse model of high-grade oligodendroglioma. Cancer Res. 74, 1833-1844 (2014).

42. Kurtsdotter, I. et al. SOX5/6/21 prevent oncogene-driven transformation of brain stem cells. Cancer Res. 77, 4985-4997 (2017).

43. Chakravarty, G. et al. Prognostic significance of cytoplasmic SOX9 in invasive ductal carcinoma and metastatic breast cancer. Exp. Biol. Med. 236, 145-155 (2011).

44. Jethon, A. et al. Prognostic significance of SOX18 expression in non-small cell lung cancer. Int. J. Oncol. 46, 123-132 (2015).

45. Wuebben, E. L. \& Rizzino, A. The dark side of SOX2: cancer - a comprehensive overview. Oncotarget 8, 44917-44943 (2017). 
46. Fan, W. et al. A novel missense mutation $224 \mathrm{G}>\mathrm{T}$ (R75M) in SRY coding region interferes with nuclear import and results in 46, XY complete gonadal dysgenesis. PLoS ONE 11, e0168484 (2016).

47. Mitchell, C. L. \& Harley, V. R. Biochemical defects in eight SRY missense mutations causing XY gonadal dysgenesis. Mol. Genet. Metab. 77, 217-225 (2002).

48. Bernard, P., Sim, H., Knower, K., Vilain, E. \& Harley, V. Human SRY inhibits beta-catenin-mediated transcription. Int. J. Biochem. Cell Biol. 40, 2889-2900 (2008).

49. Forwood, J. K., Harley, V. \& Jans, D. A. The C-terminal nuclear localization signal of the sex-determining region Y (SRY) high mobility group domain mediates nuclear import through importin beta 1. J. Biol. Chem. 276 46575-46582 (2001).

50. Gontan, C. et al. Exportin 4 mediates a novel nuclear import pathway for Sox family transcription factors. J. Cell Biol. 185, 27-34 (2009).

51. Pumroy, R. A., Ke, S., Hart, D. J., Zachariae, U. \& Cingolani, G. Molecular determinants for nuclear import of influenza A PB2 by importin alpha isoforms 3 and 7. Structure 23, 374-384 (2015).

52. Smith, K. M. et al. Structural basis for importin alpha 3 specificity of $\mathrm{W}$ proteins in Hendra and Nipah viruses. Nat. Commun. 9, 3703 (2018).

53. Roman, N., Christie, M., Swarbrick, C. M., Kobe, B. \& Forwood, J. K. Structural characterisation of the nuclear import receptor importin alpha in complex with the bipartite NLS of Prp20. PLOS ONE 8, e82038 (2013).

54. Sarker, S. et al. Structural insights into the assembly and regulation of distinct viral capsid complexes. Nat. Commun. 7, 13014 (2016).

55. Aragao, D. et al. MX2: a high-flux undulator microfocus beamline serving both the chemical and macromolecular crystallography communities at the Australian Synchrotron. J. Synchrotron Radiat. 25, 885-891 (2018).

56. Cowieson, N. P. et al. MX1: a bending-magnet crystallography beamline serving both chemical and macromolecular crystallography communities at the Australian Synchrotron. J. Synchrotron Radiat. 22, 187-190 (2015)

57. Battye, T. G., Kontogiannis, L., Johnson, O., Powell, H. R. \& Leslie, A. G. iMOSFLM: a new graphical interface for diffraction-image processing with MOSFLM. Acta Crystallogr. D Biol. Crystallogr. 67, 271-281 (2011).

58. Evans, P. R. An introduction to data reduction: space-group determination, scaling and intensity statistics. Acta Crystallogr. D Biol. Crystallogr. 67, 282-292 (2011).

59. Winn, M. D. et al. Overview of the CCP4 suite and current developments. Acta Crystallogr. D Biol. Crystallogr. 67, 235-242 (2011).

60. Marfori, M., Lonhienne, T. G., Forwood, J. K. \& Kobe, B. Structural basis of high-affinity nuclear localization signal interactions with importin-alpha. Traffic 13, 532-548 (2012).

61. Jeong, S. A. et al. Akt-mediated phosphorylation increases the binding affinity of hTERT for importin alpha to promote nuclear translocation. J. Cell Sci. 128, 2951 (2015).

62. Emsley, P. \& Cowtan, K. Coot: model-building tools for molecular graphics. Acta Crystallogr. D Biol. Crystallogr. 60, 2126-2132 (2004).

63. Adams, P. D. et al. PHENIX: a comprehensive Python-based system for macromolecular structure solution. Acta Crystallogr. D Biol. Crystallogr. 66, 213-221 (2010).

64. Zhang, Y. et al. Chromatin connectivity maps reveal dynamic promoterenhancer long-range associations. Nature 504, 306-310 (2013).

65. Barbarani, G., Fugazza, C., Barabino, S. M. L. \& Ronchi, A. E. SOX6 blocks the proliferation of BCR-ABL1(+) and JAK2V617F(+) leukemic cells. Sci. Rep. 9, 3388 (2019).

66. Cavallaro, M. et al. Impaired generation of mature neurons by neural stem cells from hypomorphic Sox2 mutants. Development 135, 541-557 (2008).
67. Hime, G. R. et al. Functional analysis in Drosophila indicates that the NBCCS/PTCH1 mutation G509V results in activation of smoothened through a dominant-negative mechanism. Dev. Dyn. 229, 780-790 (2004).

\section{Acknowledgements}

J.K.F. acknowledges support from the NHMRC Ideas Grants APP1188175. C.F.B. acknowledges support from NIH grants R21AI144880 and P01AI120943. M.S. acknowledges support from MRC grants MC-A025-5PL41, and MC_UP_1201/6 and Leverhulme Emeritus Fellowship EM-2016-062.

\section{Author contributions}

B.J. acquired and analyzed the data, M.E. acquired and analyzed the data, M.P. acquired and analyzed the data, K.M.W. acquired and analyzed the data, D.A. interpreted the data N.R. acquired and analyzed the data, J.D.N. acquired and analyzed the data, S.R.R. interpreted the data, N.D. acquired and analyzed the data, M.S. analyzed the data and revised the manuscript, D.A.J. analyzed the data and revised the manuscript, G.R.H. analyzed the data and revised the manuscript, S.K.N. analyzed the data and revised the manusript, C.F.B. analyzed the data and revised the manuscript, and J.K.F. analyzed the data and revised the manuscript.

\section{Competing interests}

The authors declare no competing interests.

\section{Additional information}

Supplementary information is available for this paper at https://doi.org/10.1038/s41467020-20194-0.

Correspondence and requests for materials should be addressed to J.K.F.

Peer review information Nature Communications thanks Gino Cingolani, and the other, anonymous, reviewer(s) for their contribution to the peer review of this work. Peer reviewer reports are available.

Reprints and permission information is available at http://www.nature.com/reprints

Publisher's note Springer Nature remains neutral with regard to jurisdictional claims in published maps and institutional affiliations.

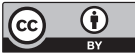

Open Access This article is licensed under a Creative Commons Attribution 4.0 International License, which permits use, sharing, adaptation, distribution and reproduction in any medium or format, as long as you give appropriate credit to the original author(s) and the source, provide a link to the Creative Commons license, and indicate if changes were made. The images or other third party material in this article are included in the article's Creative Commons license, unless indicated otherwise in a credit line to the material. If material is not included in the article's Creative Commons license and your intended use is not permitted by statutory regulation or exceeds the permitted use, you will need to obtain permission directly from the copyright holder. To view a copy of this license, visit http://creativecommons.org/ licenses/by/4.0/

(C) The Author(s) 2021 